\title{
Balance energético y capacidad gluconeogénica de vacas lecheras a pastoreo tratadas con una baja dosis de somatotropina recombinante bovina en el período de transición
}

\author{
Gluconeogenic capacity and energy balance in grazing dairy cows treated with a low dose of \\ recombinant bovine somatotropin in transition period \\ JL Sánchez ${ }^{\mathrm{a}}$, CA Wagemann ${ }^{\mathrm{b}}$, C Strieder-Barboza ${ }^{\mathrm{c}}, \mathrm{M} \mathrm{Noro}^{\mathrm{b}, \mathrm{d}^{*}}$ \\ ${ }^{a}$ Universidad La Salle, Bogotá, Colombia. \\ 'Instituto de Ciencias Clínicas Veterinarias, Facultad de Medicina Veterinaria, Universidad Austral de Chile, Valdivia, Chile. \\ 'Grupo de Investigación en Nutrición, Toxicología y Reproducción Animal, Facultad de Medicina Veterinaria y Zootecnia, \\ Universidad Cooperativa de Colombia, Bucaramanga, Colombia. \\ ${ }^{\mathrm{d} C u r s o ~ d e ~ M e d i c i n a ~ V e t e r i n a r i a, ~ U n i v e r s i d a d e ~ F e d e r a l ~ d o ~ P a m p a, ~ U N I P A M P A, ~ U r u g u a i a n a, ~ B r a s i l . ~}$
}

\begin{abstract}
SUMMARY
The aim of the study was to establish the low dose effect $(250 \mathrm{mg} / \mathrm{cow})$ of recombinant bovine somatotropin (rbST) in grazing dairy cows during the transition period on energy-protein balance parameters and gluconeogenic capacity. 3 assays were conducted: Prepartum, 10 prepartum cows were used: bST group (bST, $\mathrm{n}=5)$, single dose of $\mathrm{rbST}$ on the $26^{\text {th }}$ day (d) prepartum, and control $(\mathrm{n}=5)$; blood samples and body condition score (BCS) were obtained every $3 \mathrm{~d}$ from $26^{\text {th }} \mathrm{d}$ to $6^{\text {th }} \mathrm{d}$ prepartum. Pospartum, 10 postpartum cows were used: bST group $(\mathrm{n}=5)$, single dose of rbST on the $6^{\text {th }} \mathrm{d}$ postpartum, and control $(\mathrm{n}=5)$; blood samples, body weight $(\mathrm{BW}), \mathrm{BCS}$ and milk production were obtained and recorded every 3 days from $6^{\text {th }} \mathrm{d}$ to $27^{\text {th }} d$ postpartum. Transition, 14 prepartum cows were used: bST group $(n=7)$, treated with rbST every 14 days from $21^{\text {th }} d$ prepartum to $7^{\text {th }} d$ postpartum, and control $(\mathrm{n}=7)$; blood samples, BW and BCS score were obtained every 3 days from $21^{\text {th }} \mathrm{d}$ prepartum to $7^{\text {th }}$ d postpartum; additionally, a propionate loading test was performed ( $\mathrm{bST}, \mathrm{n}=4$; control, $\mathrm{n}=4$ ) on the $9^{\text {th }} \mathrm{d}$ postpartum to assess conversion of propionate to glucose. In all the assays, energy and protein plasma concentration were determined. The treatment of grazing dairy cows with a single dose of rbST in prepartum did not change the energy balance, while in early postpartum it increased milk production without a detrimental effect on energy balance. Propionate to glucose conversion in early postpartum was not affected by the prepartum administration of rbST.
\end{abstract}

Key words: energy-protein balance, transition period, somatotropin, cows.

\section{RESUMEN}

El objetivo del estudio fue determinar el efecto de la dosis baja $(250 \mathrm{mg} / \mathrm{vaca})$ de somatotropina recombinante bovina (rbST) en vacas lecheras a pastoreo en período de transición sobre los indicadores energético-proteínicos y capacidad gluconeogénica. Se realizaron 3 ensayos: Preparto, se utilizaron 10 vacas preparto, grupo bST ( $=5$ ): dosis única de rbST el día (d) 26 preparto, y control $(\mathrm{n}=5)$; se obtuvieron muestras de sangre y se registró la condición corporal $(\mathrm{CC})$ cada $3 \mathrm{~d}$ desde el $26 \mathrm{~d}$ al $6 \mathrm{~d}$ preparto. Posparto, se utilizaron 10 vacas posparto, grupo bST $(\mathrm{n}=5)$ : dosis única de rbST el 6 d posparto, y control ( $\mathrm{n}=5$ ); se obtuvieron muestras de sangre cada $3 \mathrm{~d}$ del $6 \mathrm{~d}$ hasta el 27 d posparto, y se determinó el peso vivo (PV), CC y producción láctea. Transición, 14 vacas preparto, grupo bST $(\mathrm{n}=7)$ : rbST cada 14 d desde el $21 \mathrm{~d}$ preparto hasta el $7 \mathrm{~d}$ posparto, y control $(\mathrm{n}=7)$; se obtuvieron muestras de sangre cada 3 d desde el 21 d preparto hasta el 7 d posparto, se registró la CC y PV y se realizó una prueba de carga de propionato el 9 d posparto ( $b S T, n=4$; control, $n=4$ ) para evaluar la conversión de propionato a glucosa. En los 3 ensayos se determinaron las concentraciones plasmáticas de indicadores energéticos-proteínicos. El tratamiento con una dosis única de rbST en el preparto no afectó el balance energético, mientras que su uso en el posparto temprano incrementó la producción láctea sin deteriorar el balance energético. La administración de rbST desde el período preparto no afectó la conversión de propionato a glucosa.

Palabras clave: balance energético-proteínico, período de transición, somatotropina, vacas.

\section{INTRODUCCIÓN}

La glucosa es el sustrato energético más importante para favorecer el metabolismo energético e incrementar la producción láctea, más aún en vacas lecheras a pasto-

Aceptado: 10.10.2013.

* mirelanoro@gmail.com reo, ya que es originada casi exclusivamente a partir de la gluconeogénesis hepática (Reynolds y col 2003). Durante el período de transición, comprendido entre las tres semanas previas al parto y las tres semanas posteriores a éste, la gluconeogénesis puede no estar totalmente estimulada. Esto se debe a una baja disponibilidad de precursores en dietas de baja calidad o a una falla en la adaptación hepática preparto, favoreciendo la presentación de balance ener- 
gético negativo (BEN) y la lipomovilización (Bruss 2008, Block 2010). El empleo de la somatotropina recombinante bovina (rbST) disminuye el uso de la glucosa por los tejidos periféricos e incrementa su demanda por el tejido mamario para la síntesis láctea; además, promueve la utilización de fuentes alternativas de energía para satisfacer los requerimientos de mantención y lactancia de las vacas lecheras sin alterar la homeostasis corporal (Drackley y col 2001, Doepel y col 2009), lo cual sugiere un efecto de estimulación de la gluconeogénesis hepática dado por la rbST. No obstante, se desconoce el efecto de una dosis única de rbST de liberación lenta en el período de transición preparto o posparto sobre el balance energético, así como el efecto del tratamiento con rbST desde el período de transición preparto sobre la capacidad gluconeogénica de vacas lecheras a pastoreo en la lactancia temprana. El objetivo del estudio fue analizar los indicadores del balance energético y la capacidad gluconeogénica vía propionato de vacas lecheras a pastoreo tratadas con dosis bajas de rbST de liberación lenta durante el período de transición.

\section{MATERIAL Y MÉTODOS}

\section{UBICACIÓN}

El estudio se realizó en el predio experimental "Santa Rosa" de la Universidad Austral de Chile (UACh), localizado en Valdivia, Chile (39 48'30" latitud sur y $73^{\circ} 14$ '30" longitud oeste), a una altura de 12 m.s.n.m, clima templado húmedo con influencia mediterránea, con una temperatura media anual de $12^{\circ} \mathrm{C}$, una precipitación de $1.871 \mathrm{~mm}$ anual, entre los meses de marzo y abril de 2011 de acuerdo a la fecha esperada de parto de las vacas.

\section{ANIMALES Y DELINEAMIENTO EXPERIMENTAL}

El estudio fue dividido en tres experimentos utilizando un total de 34 vacas Holstein-Friesian de 3 a 7 años de edad y 2 a 4 partos.

Ensayo preparto. Su objetivo fue analizar el balance energético de las vacas lecheras tratadas con una dosis única de rbST de liberación lenta en el período de transición preparto. Para esto se utilizaron 10 vacas asignadas a los $32 \pm 3$ días previo a la fecha esperada de parto por condición corporal (CC) y producción láctea de la lactancia anterior a los grupos bST $(\mathrm{n}=5 ; \mathrm{CC}=3,40 \pm 0,13)$ y control $(\mathrm{n}=5 ; \mathrm{CC}=3,50 \pm 0,29)(\mathrm{P}>0,05)$. En el día $26 \pm 3$ preparto se administró a cada vaca del grupo bST la dosis única de rbST. La obtención de las muestras de sangre y registro de la CC se realizó cada 3 días desde el día 27 hasta el día 6 preparto.

Ensayo posparto. Su objetivo fue analizar el balance energético y producción láctea de las vacas lecheras suministradas con una dosis única de rbST de liberación lenta en el período de transición posparto. Para esto se utilizaron 10 vacas, asignadas a los $14 \pm 3$ días previo a la fecha esperada de parto por CC y producción láctea de la lactancia anterior a los grupos bST $(\mathrm{n}=5 ; \mathrm{CC}=3,50$ $\pm 0,29 ; \mathrm{PV}=662 \pm 78 \mathrm{~kg})$ y control $(\mathrm{n}=5 ; \mathrm{CC}=3,75 \pm$ $0,29 ; \mathrm{PV}=693 \pm 85 \mathrm{~kg})(\mathrm{P}>0,05)$. A cada vaca del grupo bST se le administró una dosis única de rbST el día 6 \pm 2 posparto. Se obtuvieron muestras de sangre y registro de la CC y producción láctea cada 3 días desde el día 6 hasta el día 27 posparto. Adicionalmente, se registró el peso vivo de cada vaca en los días 6,18 y 30 posparto.

Ensayo transición. Su objetivo fue analizar el balance energético y capacidad gluconeogénica vía propionato de las vacas lecheras tratadas con bajas dosis de rbST en el período de transición ( 3 dosis; 1 cada 14 días: días $20 \pm 3$ y $5 \pm 2$ preparto y $6 \pm 3$ posparto). Se utilizaron 14 vacas asignadas a los $28 \pm 3$ días previo a la fecha esperada de parto por $\mathrm{CC}$ y producción láctea de la lactancia anterior a los grupos bST $(n=7 ; C C=3,75 \pm 0,45)$ y control $(n=7$; $\mathrm{CC}=3,50 \pm 0,27$ ). Se obtuvieron muestras de sangre $\mathrm{y}$ registro de la CC cada 3 días desde el día $21 \pm 2$ preparto hasta el día $6 \pm 2$ posparto; el peso vivo de cada vaca se registró en el último día experimental. Para la determinación de la capacidad gluconeogénica (día $9 \pm 2$ posparto) se seleccionaron aleatoriamente 8 vacas $(\mathrm{bST}=4 ; \mathrm{C}=4)$, a las cuales se les realizó una prueba de carga de propionato de sodio ( $\geq 99,0 \%$, Sigma-Aldrich Corp.) (Grohn 1985) a través de un catéter estéril (Certofix ${ }^{\circledR}$ Mono S 420, 14G, B. Braun) fijado en la vena yugular izquierda o derecha. La prueba consistió en la obtención de dos muestras de sangre, a los -5 y 0 minutos previos a la infusión (valores basales), seguidas de la infusión de un bolo de propionato de sodio $(2,5 \mathrm{mM} / \mathrm{kg}$ pv a una concentración de 1,84 M, $\mathrm{pH} 7,4)$ en un lapso de $2,5 \pm 0,5$ minutos. Finalmente se obtuvieron muestras de sangre a los 5, 10, 15, 20, 30, 40, 50, 60, 75 y 90 minutos posteriores a la infusión.

Las dosis de rbST utilizadas en los 3 ensayos (Boos$\operatorname{tin}^{\circledR}-250,250 \mathrm{mg} / 14$ días, $2 \mathrm{~mL}$, LG Life Sciences) fueron administradas en la fosa isquiorrectal previo a la ración de la mañana en el preparto y después del ordeño de la mañana en las vacas posparto. Los animales del grupo control no recibieron tratamiento alguno.

\section{MANEJO Y DIETA DE LOS ANIMALES}

En el período preparto (ensayo preparto y de transición) las vacas de ambos grupos fueron mantenidas en un potrero con bajo aporte de pradera (<10 kg MS/vaca/día), y la ración fue entregada en comederos colectivos dos veces al día (8:00 y 16:00 horas). La dieta consistió de heno de pradera ad libitum, $4 \mathrm{~kg} / \mathrm{vaca} /$ día de concentrado (18\% P; 3,0 Mcal EM/kg MS; 45\% CNF; 25\% FDN) y $0,15 \mathrm{~kg}$ vaca/día de sales aniónicas.

En el posparto (ensayo posparto y ensayo transición) las vacas fueron manejadas en un único grupo, pastorean- 
do pradera de Lolium perenne con una oferta aproximada de $20 \mathrm{~kg}$ MS/vaca/día. Además ,recibieron en comederos colectivos una ración constituida por heno de trigo ad libitum, $5 \mathrm{~kg}$ de concentrado/vaca/día (88\% MS; 20\% PB; $35 \%$ PNDR; $6 \%$ FC; $20 \%$ FDN; $51 \%$ CS; 38\% almidón; $3 \mathrm{Mcal}$ EM/kg MS) y $5 \%$ de mezcla mineral, distribuida en dos raciones equitativas posterior a las ordeñas (6:00 y 16:00 horas).

\section{OBTENCIÓN Y ANÁLISIS DE LAS MUESTRAS}

Las muestras de sangre de cada vaca fueron obtenidas mediante venopunción coccígea, utilizando agujas Vacutainer $^{\circledR}(2,54 \mathrm{~cm}, 21 \mathrm{G})$, previo a la administración de rbST y depositadas en tubos con heparina sódica (10x 100 $\mathrm{mm}$ ) y fluoruro de sodio $(10 \times 100 \mathrm{~mm})$. Una vez en el laboratorio, fueron centrifugadas a $3.000 \mathrm{~g}$ por $10 \mathrm{mi}-$ nutos (Beckman ${ }^{\circledR}$ TJ6) para la obtención del plasma, el cual fue transferido a microtubos de $1,5 \mathrm{~mL}$ rotulados y almacenados a $-20^{\circ} \mathrm{C}$ para su posterior análisis. A partir de las muestras de plasma con heparina sódica se determinaron las concentraciones de ácidos grasos no esterificados (NEFA; ACS-ACOD, Wako ${ }^{\circledR}$ ), $\beta$-hidroxibutirato ( $\beta$ HBA; 3-HBDH, Randox $\left.{ }^{\circledR}\right)$, colesterol (CHOD-PAP) y albúmina (BCG, Human ${ }^{\circledR}$ ), y la actividad de la glutamato deshidrogenasa (GMD, EC 1.4.1.2; Diasys ${ }^{\circledR}$ ). En las muestras de plasma con fluoruro de sodio se determinó la concentración de glucosa (GOD-PAP, Human ${ }^{\circledR}$ ). Para los procedimientos mencionados se utilizó un espectrofotómetro automático Metrolab 2300 (Wiener $\mathrm{Lab}^{\circledR}$ ).

Los datos de producción láctea diaria del ensayo posparto fueron registrados en medidores de leche tipo Waikato de $42 \mathrm{~kg}$ (Waikato ${ }^{\circledR}$ ), instalados en una sala de ordeño tipo tándem. La CC de cada vaca fue registrada considerando la escala de 1 a $5(1=$ delgada, $5=$ obesa $)$ a intervalos de 0,25 puntos (Ferguson y col 1994) y el peso (PV) se determinó mediante una báscula romana fija. El cálculo de las pendientes de CC y de PV se realizó usando modelos de regresión lineal.

\section{ANÁLISIS ESTADÍSTICO}

Los datos de los tres ensayos fueron analizados en cuanto a normalidad y homocedasticidad mediante Shapiro-Wilk y la prueba de Bartlett, respectivamente, usando el programa estadístico Statistix 8.0 (Statistix 2003). Los tratamientos se compararon mediante un modelo linear generalizado de ANDEVA, considerando los períodos pre y posparto por separado en el ensayo transición. Se utilizó el modelo $\mathrm{Y}_{\mathrm{ij}}=\mu+\mathrm{T}_{\mathrm{i}}+\mathrm{D}_{\mathrm{j}}+\mathrm{TD}_{\mathrm{ij}}+\varepsilon_{\mathrm{ij}}$ para analizar los datos donde: $\mathrm{Y}_{\mathrm{ij}}=$ el efecto calculado, $\mu=$ media general, $\mathrm{T}_{\mathrm{i}}=$ efecto del i-ésimo tratamiento, $\mathrm{D}_{\mathrm{j}}=$ efecto del j-ésimo día, $\mathrm{TD}_{\mathrm{ij}}=$ interacción tratamiento y día y $\varepsilon_{\mathrm{ij}}=$ error. Aquellas variables que no siguieron una distribución normal fueron analizadas mediante un test de Kruskal-Wallis. Las pendientes de PV y CC se compararon usando la prueba de $t$ de Student o prueba de $U$ (Mann-Whitney) cuando los datos presentaron una distribución no paramétrica. A partir de los datos obtenidos en la prueba de carga de propionato de sodio se calculó el área bajo la curva para la glucosa $\left(\mathrm{ABC}_{\mathrm{GLU} 90}\right)$, usando un modelo trapezoidal que consideró el tiempo transcurrido desde el inicio de la infusión de propionato hasta los 90 minutos posteriores a esta, y utilizando como valor basal la media de las concentraciones de los tiempos -5 y 0 (Bradford y col 2006). Para todos los análisis se consideró una diferencia significativa cuando $\mathrm{P}<0,05$.

\section{RESULTADOS Y DISCUSIÓN}

\section{ENSAYO PREPARTO}

Las concentraciones medias de NEFA y albúmina, la actividad de GMD y la pendiente de CC fueron similares entre los grupos ( $\mathrm{P}>0,05$; cuadro 1). Por otro lado, la

Cuadro 1. Valores de indicadores energético-proteínicos $(\bar{x} \pm \mathrm{DE})$ en vacas lecheras tratadas con una dosis única de rbST en el período de transición preparto.

Values of energy-protein indicators $(\bar{x} \pm \mathrm{SD})$ in dairy cows treated with a single dose of rbST during pre partum transition period.

\begin{tabular}{lcc}
\hline Variable & bST (IC) & Control (IC) \\
\hline NEFA $(\mu \mathrm{mol} / \mathrm{L})$ & $353 \pm 170(204-502)$ & $409 \pm 137(290-528)$ \\
$\beta$ HBAA $(\mathrm{mmol} / \mathrm{L})$ & $0,32 \pm 0,06(0,26-0,38)$ & $0,54 \pm 0,06(0,48-0,60)^{*}$ \\
Glucosa $(\mathrm{mmol} / \mathrm{L})$ & $3,38 \pm 0,20(3,21-3,55)$ & $3,02 \pm 0,20(2,85-3,19)^{*}$ \\
Colesterol $(\mathrm{mmol} / \mathrm{L})$ & $3,43 \pm 0,20(3,26-3,60)$ & $3,13 \pm 0,20(2,96-3,30)^{*}$ \\
Albúmina $(\mathrm{g} / \mathrm{L})$ & $39,4 \pm 1,12(38,4-40,4)$ & $39,2 \pm 1,12(38,2-40,2)$ \\
GMD $(\mathrm{U} / \mathrm{L})$ & $27,7 \pm 8,74(20,1-35,3)$ & $20,0 \pm 8,74(12,4-27,6)$ \\
CC $(1-5)^{*}$ & $0,021 \pm 0,01(0,008-0,034)$ & $-0,056 \pm 0,01(-0,176-0,064)$ \\
\hline
\end{tabular}

*Pendiente de condición corporal semanal. IC= intervalo de confianza. $* \mathrm{P}<0,05$. 
concentración de $\beta \mathrm{HBA}$ fue superior en el grupo control, mientras que las de glucosa y colesterol fueron mayores en el grupo bST ( $\mathrm{P}<0,05$; cuadro 1$)$. Si bien las concentraciones medias de NEFA fueron similares en ambos grupos, en el grupo control se encontraron por sobre el límite superior de referencia $(<400 \mu \mathrm{mol} / \mathrm{L}$; Cuadro 1$)$, lo que asociado a las mayores concentraciones de $\beta \mathrm{HBA}$ en este grupo sugieren un incremento de la lipomovilización causada por un BEN debido a la mayor demanda energética decurrente de la gestación (Block 2010). Por otro lado, la mayor glucemia y menor concentración de $\beta \mathrm{HBA}$ en el grupo bST indicó un mejor balance energético (Prado y col 2003), lo que podría asociarse a una mayor capacidad gluconeogénica o mayor ingesta de materia seca (IMS), siendo esto último reflejado en la mayor colesterolemia $(\mathrm{P}<0,05)$ (Kim y Suh 2003).

\section{ENSAYO POSPARTO}

Las concentraciones promedio de NEFA, $\beta$ HBA, glucosa, colesterol y albúmina, así como la actividad de la GMD y las pendientes de CC y PV, fueron similares entre grupos ( $\mathrm{P}>0,05$; cuadro 2). Sin embargo, la producción láctea del grupo bST fue mayor (3,6 L/vaca/día; $\mathrm{P}<0,05)$ que la del grupo control (cuadro 2). Por otro lado, vaquillas tratadas con bST en el preparto, tuvieron una menor pérdida de CC y una menor movilización lipídica, en el posparto temprano, a pesar de que produjeron más leche que las no tratadas (Schneider y col 2012). Los resultados sugieren que la administración de la dosis única de rbST en el período de transición posparto genera un incremento en la producción láctea sin afectar negativamente el metabolismo energético-proteínico.

\section{ENSAYO TRANSICIÓN}

En el preparto se observó en el grupo bST una mayor concentración plasmática de NEFA, y menor glucemia ( $\mathrm{P}<0,05$; cuadro 3 ), sin embargo, las concentraciones de $\beta$ HBA, colesterol y albúmina, así como las pendientes de CC y PV, fueron similares entre grupos ( $\mathrm{P}>0,05$; cuadro 3 ). La mayor concentración de NEFA en el grupo bST difirió de lo obtenido en el ensayo preparto. Esto probablemente estuvo asociado al mayor número de dosis de rbST empleadas y al hecho de que en este ensayo se consideró la semana del parto, momento en que las concentraciones de NEFA son máximas (Van Saun 2010). Por otro lado, contrario a los resultados del ensayo preparto, las concentraciones de $\beta$ HBA fueron similares $(\mathrm{P}>0,05)$ y se mantuvieron dentro de los límites de referencia en ambos grupos, lo que en conjunto con las concentraciones de NEFA, también dentro de los valores referenciales, indican un adecuado balance energético en el preparto (Block 2010).

Durante el período posparto todos los indicadores energéticos y proteínicos presentaron valores similares entre los grupos ( $\mathrm{P}>0,05$; cuadro 3 ). A su vez, en el grupo control se observaron concentraciones de $\beta \mathrm{HBA}$ por sobre el límite superior de referencia $(<0,6 \mathrm{mmol} / \mathrm{L}$; cuadro 3), indicando un BEN. Por otro lado, en el grupo bST la actividad de GMD se encontró por sobre el límite de referencia, lo que sugiere una leve hepatolipidosis (Bobe y col 2004, Block 2010) o micotoxicosis (Coppock 1986).

Las concentraciones de glucosa a los 90 minutos de la infusión de propionato de sodio así como el $\mathrm{ABC}_{\mathrm{GLU} 90}$, fueron similares entre los grupos ( $\mathrm{P}>0,05$; figura 1$)$, indicando que el tratamiento con bajas dosis de rbST desde

Cuadro 2. Valores de indicadores energético-proteínicos $(\bar{x} \pm \mathrm{DE})$ en vacas lecheras tratadas con una dosis única de rbST en el período de transición posparto.

Values of energy-protein indicators $(\bar{x} \pm \mathrm{SD})$ in dairy cows treated with a single dose of rbST during post partum transition period.

\begin{tabular}{lcc}
\hline Variable & bST (IC) & Control (IC) \\
\hline NEFA $(\mu \mathrm{mol} / \mathrm{L})$ & $355 \pm 90(276-433)$ & $308 \pm 90(229-387)$ \\
BHBA $(\mathrm{mmol} / \mathrm{L})$ & $0,83 \pm 0,15(0,69-0,97)$ & $0,84 \pm 0,15(0,70-0,98)$ \\
Glucosa $(\mathrm{mmol} / \mathrm{L})$ & $2,96 \pm 0,10(2,86-3,06)$ & $3,11 \pm 0,10(3,01-3,21)$ \\
Colesterol $(\mathrm{mmol} / \mathrm{L})$ & $3,54 \pm 0,42(3,17-3,91)$ & $3,34 \pm 0,42(2,97-3,71)$ \\
Albúmina $(\mathrm{g} / \mathrm{L})$ & $38,9 \pm 1,70(37,3-40,5)$ & $37,6 \pm 1,70(36,0-39,2)$ \\
GMD $(\mathrm{U} / \mathrm{L})$ & $19,6 \pm 5,40(14,3-24,9)$ & $24,5 \pm 5,40(19,2-29,8)$ \\
Pend CC $(1-5)^{*}$ & $-0,021 \pm 0,06(-0,08-0,04)$ & $-0,049 \pm 0,06(-0,11-0,01)$ \\
Pend Peso vivo $(\mathrm{kg})^{\dagger}$ & $3,9 \pm 7,0(-3,35-11,1)$ & $2,2 \pm 7,0(-5,05-9,45)$ \\
P láctea (L/vaca/día) & $25,4 \pm 1,9(23,6-27,2)$ & $21,8 \pm 1,8(24,2-23,4)^{*}$ \\
\hline
\end{tabular}

*Pendiente de condición corporal; 'Pendiente de peso vivo; "Producción láctea. IC= intervalo de confianza. * P < 0,05. 
Cuadro 3. Valores de indicadores energético-proteínicos $(\bar{x} \pm \mathrm{DE})$ y área bajo la curva de glucosa a los 90 minutos de la infusión de propionato de sodio en el posparto en vacas tratadas con bajas dosis de rbST en el período de transición preparto y posparto.

Values of energy-protein indicators $(\bar{x} \pm \mathrm{SD})$ and area under the glucose curve after 90 minutes of infusion of sodium propionate in the postpartum period in cows treated with low doses of rbST during pre and post partum transition period.

\begin{tabular}{|c|c|c|c|c|}
\hline \multirow[t]{2}{*}{ Variable } & \multicolumn{2}{|c|}{ Preparto } & \multicolumn{2}{|c|}{ Posparto } \\
\hline & bST (IC) & Control (IC) & bST (IC) & Control (IC) \\
\hline NEFA $(\mu \mathrm{mol} / \mathrm{L})$ & $237 \pm 65(188-286)$ & $160 \pm 70(107-212)^{*}$ & $441 \pm 146(332-550)$ & $386 \pm 151(272-500)$ \\
\hline$\beta \mathrm{HBA}(\mathrm{mmol} / \mathrm{L})$ & $0,39 \pm 0,05(0,35-0,43)$ & $0,37 \pm 0,05(0,33-0,41)$ & $0,51 \pm 0,10(0,43-0,59)$ & $0,66 \pm 0,18(0,58-0,74)$ \\
\hline Glucosa (mmol/L) & $3,38 \pm 0,13(3,28-3,47)$ & $3,62 \pm 0,13(3,52-3,72)^{*}$ & $3,35 \pm 0,23(3,17-3,53)$ & $3,26 \pm 0,23(3,08-3,44)$ \\
\hline Colesterol (mmol/L) & $3,12 \pm 0,18(2,98-3,26)$ & $2,92 \pm 0,21(2,76-3,08)^{\#}$ & $2,49 \pm 0,36(2,22-2,76)$ & $2,57 \pm 0,39(2,28-2,86)$ \\
\hline Albúmina (g/L) & $39,0 \pm 1,56(37,8-40,2)$ & $39,5 \pm 1,56(38,3-40,7)$ & $39,9 \pm 1,82(39,8-40,0)$ & $39,4 \pm 2,08(39,2-39,6)$ \\
\hline GMD (U/L) & $25,2 \pm 7,0(19,9-30,5)$ & $22,5 \pm 7,5(16,8-28,2)$ & $34,3 \pm 16,9(21,6-47,0)$ & $19,8 \pm 5,5(15,7-23,9)$ \\
\hline Pend CC $(1-5)^{*}$ & $-0,02 \pm 0,08(-0,08-0,04)$ & $0,02 \pm 0,08(-0,04-0,08)$ & $-0,12 \pm 0,29(-0,34-0,10)$ & $-0,05 \pm 0,32(-0,28-0,19)$ \\
\hline Pend Peso vivo $(\mathrm{kg})^{\dagger}$ & -- & -- & $3,2 \pm 12,0(-5,8-12,2)$ & $-7,9 \pm 20,3(-23,2-7,4)$ \\
\hline Glucosa propionato $(\mathrm{mmol} / \mathrm{L})^{\ddagger}$ & -- & -- & $4,09 \pm 0,26(3,89-4,29)$ & $4,07 \pm 0,26(3,87-4,27)$ \\
\hline $\mathrm{ABC}_{\mathrm{GLU} 90}$ & -- & -- & $72,1 \pm 62,4(24,9-119,3)$ & $56,8 \pm 14,8(45,6-67,9)$ \\
\hline
\end{tabular}

* Pendiente de condición corporal; ${ }^{\dagger}$ Pendiente de peso vivo; ${ }^{\ddagger}$ Concentración promedio de glucosa luego de la prueba de carga de propionato; ${ }^{+}$Área bajo la curva de glucosa a los 90 minutos de la infusión de propionato de sodio. $\mathrm{IC}=$ intervalo de confianza. * $\mathrm{P}<0,05$. "P $<0,10 \mathrm{y}>0,05$.

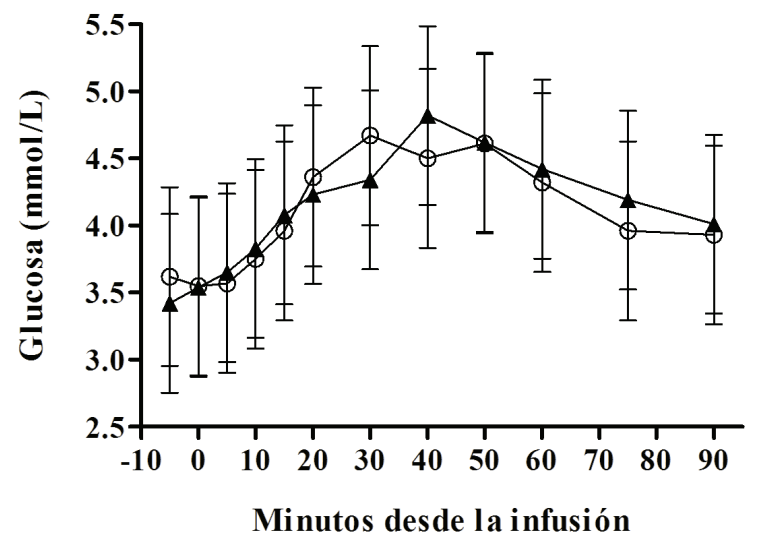

Figura 1. Concentración $(\bar{x} \pm \mathrm{DE})$ de glucosa desde los minutos -5 a 90 post infusión de propionato en vacas lecheras tratadas con dosis bajas de rbST $(\boldsymbol{\Delta})$ y vacas controles $(\bigcirc)$ a los $9 \pm 2$ días posparto.

Concentration $(\bar{x} \pm \mathrm{SD})$ of glucose from -5 to 90 minutes post infusion of propionate in dairy cows treated with low doses of $\operatorname{rbST}(\Delta)$ and control cows $(\bigcirc)$ at $9 \pm 2$ days postpartum.

el preparto no estimuló la capacidad gluconeogénica vía propionato en el posparto. Se ha reportado que la rbST estimula la gluconeogénesis hepática asociada al incremento en el uso de precursores glucogénicos y a la baja respuesta a la insulina que, por otro lado, incrementan la actividad de la enzima gluconeogénica fosfoenolpiruva- to carboxiquinasa (PEPCK, EC 4.1.1.32) (Velez y Donkin 2005). El hecho de que en el presente ensayo no se observó un incremento en la conversión de propionato a glucosa podría asociarse a que en el período de transición posparto la gluconeogénesis a partir de otros precursores glucogénicos, como alanina, lactato y glicerol (Larsen y Kristensen 2009) adquiere mayor importancia que el propionato para sostener la demanda de glucosa hacia la glándula mamaria; sin embargo, éstos no fueron evaluados en el estudio.

Se concluye que el tratamiento con una dosis baja única de rbST en vacas lecheras a pastoreo no afectó el balance energético cuando administrada en el preparto, mientras que su uso en el posparto temprano incrementó la producción láctea sin deteriorar el balance energético.

La administración de la dosis baja rbST desde el período de transición preparto no afectó la conversión de propionato a glucosa en el posparto temprano.

\section{AGRADECIMIENTOS}

Los autores agradecen a ANASAC por financiar el estudio y al Laboratorio de Patología Clínica Veterinaria de la Universidad Austral de Chile por su asistencia e invaluable colaboración.

\section{REFERENCIAS}

Block E. 2010. Transition cow research - What makes sense today? Proceedings High Plains Dairy Conference, Ama- 
rillo, Texas, USA, Pp 75-98.

Bobe G, JW Young, DC Beitz. 2004. Invited review: pathology, etiology, prevention, and treatment of fatty liver in dairy cows. J Dairy Sci 87, 3105-3124.

Bradford BJ, AD Gour, AS Nash, MS Allen. 2006. Propionate challenge tests have limited value for investigating bovine metabolism. J Nutr 136, 1915-1920.

Bruss ML. 2008. Lipids and Ketones. In: Kaneko JJ, Harvey JW, Bruss ML (eds). Clinical Biochemistry of Domestic Animals. Academic Press San Diego, California, USA, Pp 81-115.

Coppock CE. 1986. Mineral utilization by the lactating cowchloride. J Dairy Sci 69, 595-603.

Doepel L, GE Lobley, JF Bernier, P Dubreuil, H Lapierre. 2009. Differences in splanchnic metabolism between late gestation and early lactation dairy cows. J Dairy Sci 92, 3233-3243.

Drackley JK, TR Overton, GN Douglas. 2001. Adaptations of glucose and long-chain fatty acid metabolism in liver of dairy cows during the periparturient period J Dairy Sci 84, E100-E112.

Ferguson JD, DT Galligan, N Thomsen. 1994. Principal descriptors of body condition score in Holstein cows. J Dairy Sci 77, 2695-2703.

Grohn Y. 1985. Propionate loading test for liver function in spontaneously ketotic dairy cows. Res Vet Sci 39, 24-28.

Kim IH, GH Suh. 2003. Effect of the amount of body condition loss from the dry to near calving periods on the subsequent body condition change, occurrence of postpartum diseases, metabolic parameters and reproductive performance in Holstein dairy cows. Theriogenology 60, 1445-1456.

Larsen M, NB Kristensen. 2009. Effect of abomasal glucose infusion on splanchnic amino acid metabolism in periparturient dairy cows. J Dairy Sci 92, 3306-3318.

Prado IN, WG Nascimento, JA Negrao, LP Rigolon, S De Souza, ML Doi Sakuno, GL Pessini. 2003. Recombinant bovine somatotropin (rBST) on haematologic aspects and metabolites of heifers ( $1 / 2$ Nellore x $1 / 2$ Red Angus) blood, in feedlot. $R$ Bras Zootec 32, 465-472.

Reynolds CK, PC Aikman, B Lupoli, DJ Humphries, DE Beever. 2003. Splanchnic metabolism of dairy cows during the transition from late gestation through early lactation. $J$ Dairy Sci 86, 1201-1217.

Schneider A, E Schwegler, P Montagner, LT Hax, E Schmitt, LFM Pfeifer, FAB Del Pino, I Bianchi, GR Paludo, MN Corrêa. 2012. Effect of prepartum somatotropin injection in late-pregnant Holstein heifers on metabolism, milk production and postpartum resumption of ovulation. Animal 6, 935-940.

Statistix. 2003. Statistix 8.0: User's manual. Tallahasse, FL, USA.

Van Saun RJ. 2010. Indicators of dairy cow transition risks: metabolic profiling revisited. In: Wittwer F, Chihuailaf R, Contreras H, Gallo C, Kruze J, Lanuza F, Letelier C, Monti G, Noro M (eds). Updates on Ruminant Production and Medicine. Andros Impresiones, Santiago, Chile, Pp 65-77.

Velez JC, SS Donkin. 2005. Feed restriction induces pyruvate carboxylase but not phosphoenolpyruvate carboxykinase in dairy cows. J Dairy Sci 88, 2938-2948. 